\title{
Disseminated Intravascular Coagulation in Newborn
}

National Cancer Institute

\section{Source}

National Cancer Institute. Disseminated Intravascular Coagulation in Newborn. NCI

Thesaurus. Code C111856.

A clotting condition characterized as a disruption in the homeostatic balance of the coagulation and fibrinolytic systems presenting as a pathological activation of coagulation mechanisms leading to the formation of small clots inside the blood vessels throughout the body of the newborn. 